\title{
SOME PATHOLOGY INVOLVING PSEUDO l-GROUPS AS GROUPS OF DIVISIBILITY
}

\author{
JORGE MARTINEZ
}

\begin{abstract}
In a partially ordered abelian group $G$, two elements $a$ and $b$ are pseudo-disjoint if $a, b \geqq 0$ and either one is zero, or both are strictly positive and each $o$-ideal which is maximal with respect to not containing $a$ contains $b$, and vice versa. $G$ is a pseudo latticegroup if every element of $G$ can be written as a difference of pseudodisjoint elements.

We prove the following theorem: suppose $G$ is an abelian pseudo lattice-group; if there is an $x>0$ and a finite set of pairwise pseudodisjoint elements $x_{1}, x_{2}, \cdots, x_{k}$ all of which exceed $x$, and in addition this set is maximal with respect to the above properties, then $G$ is not a group of divisibility.

The main consequence of this result is that every so-called " $v$ group" $V\left(\Lambda, R_{\lambda}\right)$ for a given partially ordered set $\Lambda$, and where $R_{\lambda}$ is a subgroup of the additive reals in their usual order, is a group of divisibility only if $\Lambda$ is a root system, and hence $V\left(\Lambda, R_{\lambda}\right)$ is a lattice-ordered group. We do give examples of pseudo lattice-groups which are not lattice-groups, and yet are groups of divisibility.

Finally, we compute for each integral domain $D$ whose group of divisibility is a lattice-group, the group of divisibility of the polynomial ring $D[x]$ in one variable.
\end{abstract}

1. Preliminaries. All groups in this paper are abelian, and in additive notation unless otherwise indicated. An integral domain here shall be a commutative ring with identity and no zero divisors. If $D$ is an integral domain and $K$ is its quotient field, then the group of divisibility of $D$ is the multiplicative group of nonzero elements of $K$ modulo the group $U(D)$ of units of $D$; in symbols $G(D) \simeq K^{*} / U(D)$. This group can be given a directed partial order by setting $x U(D) \leqq y U(D)$ if $y x^{-1} \in D$. A (directed) p.o. group $G$ is called a group of divisibility if there is an integral domain $D$ such that $G \simeq G(D)$. We can also view this concept in terms of semivaluations: let $K$ be a field, $G$ be a directed p.o. group, and $v: K^{*} \rightarrow G$

Presented to the Society, June 27, 1972 under the title Some pathology in connection with groups of divisibility; received by the editors August 28, 1972.

AMS (MOS) subject classifications (1970). Primary 06A60, 13G05, 13H99, 13 F20.

Key words and phrases. Group of divisibility, semivaluation, pseudo-disjointness, pseudo l-group, $v$-group $V\left(\Lambda, R_{\lambda}\right)$, primitive polynomial. 
be a mapping onto $G$ satisfying

(i) $v(x y)=v(x)+v(y)$, for all $x, y \in K^{*}$;

(ii) $v(-1)=0$;

(iii) $v(x+y) \geqq g$ if $v(x), v(y) \geqq g$, with $x, y \in K^{*}$ and $g \in G$.

Such a mapping is called a semivaluation. Let $D=\left\{x \in K^{*} \mid v(x) \geqq 0\right\}$; then $D$ is a subring of $K, K$ is its quotient field and $G \simeq G(D)$. Conversely, if $D$ is an integral domain and $K$ is its quotient field, then the canonical mapping $K^{*} \rightarrow G(D)$ is a semivaluation (see [5, p. 8]; also [9, p. 1148]).

Consequently, $G$ is a group of divisibility if and only if there is a semivaluation onto $G$.

If $G$ is a totally ordered group (abbreviation $o$-group), the map $v$ is called a valuation, and Krull [6, p. 164] demonstrated that every 0 -group is a group of divisibility. Jaffard [4, p. 264] then showed that all latticegroups (abbreviation l-groups) are groups of divisibility.

In a p.o. group a directed, convex subgroup is called an o-ideal. Suppose $G$ is a p.o. group and $0 \leqq a, b \in G ; a$ and $b$ are pseudo-disjoint if either is zero, or both are strictly positive, and every $o$-ideal which is maximal with respect to not containing $a$ contains $b$, and vice versa. A pseudo lattice-group (abbreviation pseudo l-group) is a p.o. group in which every element can be written as the difference of two pseudo-disjoint elements. For the basic material concerning pseudo l-groups we refer the reader to [1] and [3]. Conrad shows in [1] that in a pseudo $l$-group $G, 0 \leqq a, b \in G$ are pseudo-disjoint if and only if $c \leqq a, b$ implies that $n c \leqq a, b$ for each positive integer $n$.

For a given partially ordered set $\Lambda$, and each $\lambda \in \Lambda$, let $R_{\lambda}$ be a subgroup of the additive real numbers equipped with the usual order. Form $V\left(\Lambda, R_{\dot{\lambda}}\right)$ : the subgroup of the cartesian product of the $R_{\lambda}$ over $\Lambda$ consisting of the "vectors" $v=\left(\cdots, v_{\lambda}, \cdots\right)$ whose supports have no infinite ascending chains. $V\left(\Lambda, R_{\lambda}\right)$ becomes a p.o. group by setting $0<v=(\cdots, v, \cdots)$ if $v_{\lambda}>0$ for each maximal component $\lambda$ of the support of $v$. Then $V\left(\Lambda, R_{\lambda}\right)$ is a pseudo l-group (see Theorem 4.8 in [1]), and every pseudo l-group may be embedded in some $V\left(\Lambda, R_{\lambda}\right)$ so as to preserve pseudo-disjointness (see 4.11 in [1]). It is well known that $V\left(\Lambda, R_{\lambda}\right)$ is an l-group if and only if $\Lambda$ is a root system: $\left\{\lambda \in \Lambda \mid \lambda \geqq \lambda_{0}\right\}$ is a chain for each $\lambda_{0} \in \Lambda$. Finally, two elements $0<v, w \in V\left(\Lambda, R_{\lambda}\right)$ are pseudo-disjoint if and only if no maximal component of the support of $v$ is comparable to one in the support of $w[\mathbf{1}$, p. 214].

2. The main theorem. We state our main result at the outset.

TheOREM A. Suppose $G$ is a pseudo l-group, and there is an element $0<x \in G$ and a set $x_{1}, x_{2}, \cdots, x_{k}$ of pairwise pseudo-disjoint elements all of which exceed $x$, and suppose further that this set is maximal with respect to the above properties. Then $G$ is not a group of divisibility. 
The proof depends on two lemmas, one rather interesting in its own right, the other rather technical.

Lemma 1. Suppose $G$ is a pseudo l-group, and $v$ is a semivaluation from a field $K$ upon $G$. If $0<a, b \in G$ are pseudo-disjoint and $0<c<a$, $b$, then there is an element $0<g \in G$, pseudo-disjoint to $a$ and $b$, with $c<g$.

Proof. Let $v(x)=a, v(y)=b$ and $g=v(x+y)$. If $c \leqq a, g$ then $c \leqq v(-x)$, so that $b=v(y)=v(x+y-x) \geqq c$. But $a$ and $b$ are pseudo-disjoint and hence $n c \leqq a, b$, for any positive integer $n$. Again using one of the defining properties of semivaluations $n c \leqq g$. Conclusion: $a$ and $g$ are pseudodisjoint; likewise $b$ and $g$ are pseudo-disjoint. It is clear that if $c<a, b$ then $c<g$; in particular $g>0$.

If $G$ is a pseudo $l$-group and $0 \neq x \in G$ we call an $o$-ideal $M$ of $G$ which is maximal with respect to not containing $x$ a value of $x$. In this language then, $a$ is pseudo-disjoint to $b$ if and only if every value of $a$ contains $b$, and vice versa.

Lemma 2. Suppose $G$ is a pseudo l-group and $0<a \in G, 0<b_{i} \in G$ $(i=1, \cdots, k)$. Assume further that the $b_{i}$ are pairwise pseudo-disjoint, while $a$ is pseudo-disjoint to $b_{1}+b_{2}+\cdots+b_{k}$. Then a is pseudo-disjoint to each $b_{i}$.

Proof. Let $M$ be a value of $a$; then by our assumption $b_{1}+b_{2}+$ $\cdots+b_{k}$ is in $M$, and so by convexity each $b_{i} \in M$. On the other hand if $N$ is a value of $b_{i}$, each $b_{j} \in N$, for $j \neq i$; this makes $N$ a value of $b_{1}+b_{2}+$ $\cdots+b_{k}$, and hence $a \in N$. It follows then that each $b_{i}$ is pseudo-disjoint to $a$.

Proof of Theorem A. Suppose $G$ is a pseudo $l$-group, $0<x \in G$ and $x_{1}, x_{2}, \cdots, x_{k}$ is a maximal, pairwise pseudo-disjoint set of elements of $G$ exceeding $x$. Relabel $x_{1}=a$ and $b=x_{2}+x_{3}+\cdots+x_{k}$; then $a$ and $b$ are pseudo-disjoint.

If $G$ is a group of divisibility as well, there is semivaluation $v$ from a field $K$ onto $G$. By Lemma 1 we may find $0<g \in G$ pseudo-disjoint to both $a$ and $b$, such that $x<g$. By Lemma $2 g$ is pseudo-disjoint to each $x_{i}(i=1, \cdots, k)$; this contradicts the maximality of the set $x_{1}, x_{2}, \cdots, x_{k}$ over $x$.

This proves the theorem.

Our first corollary concerns $v$-groups.

THEOREM B. Let $\Lambda$ be a partially ordered set, $R_{\lambda}$ be an ordered subgroup of the reals for each $\lambda \in \Lambda$; set $V=V\left(\Lambda, R_{\lambda}\right)$. If $V$ is a group of divisibility then $\Lambda$ is a root system and hence $V$ is an l-group. 
Proof. If $\Lambda$ is not a root system there exists a $\nu \in \Lambda$ with pairwise incomparable elements above $\nu$ in $\Lambda$. Let $\left\{\lambda_{i} \mid i \in I\right\}$ be a set of mutually incomparable elements of $\Lambda$ all of which exceed $\nu$, and suppose $\left\{\lambda_{i} \mid i \in I\right\}$ is also maximal with respect to these properties. Fix $j \in I$ and define $a, b \in V$ as follows:

$$
\begin{aligned}
& a_{\lambda}=1, \quad \text { if } \lambda=\lambda_{j}, \quad b_{\lambda}=1, \quad \text { if } \lambda=\lambda_{i}, i \neq j, \\
& =0 \text {, otherwise; } \quad=0 \text {, } \text { otherwise. }^{1}
\end{aligned}
$$

Clearly $0<a, b \in V$ and $a$ is pseudo-disjoint to $b$; moreover the pair $\{a, b\}$ satisfies the conditions of Theorem $\mathrm{A}$ relative to, say, $x \in V$, where

$$
\begin{array}{rlr}
x_{\lambda}=1 & \text { if } \lambda=v \\
=0 & & \text { otherwise }
\end{array}
$$

By the theorem we obtain a contradiction: for if there is an element $0<$ $g \in G$, pseudo-disjoint to both $a$ and $b$ which exceeds $x$, then we contradict the maximality of the set $\left\{\lambda_{i} \mid i \in I\right\}$ over $\nu$. Thus $V$ cannot be a group of divisibility unless $\Lambda$ is a root system.

If $G$ is a pseudo $l$-group and $0<u \in G$ has the property that no strictly positive element is pseudo-disjoint to $u$, we call $u$ a weak order unit.

Corollary 1. Suppose the pseudo l-group $G$ has a weak order unit $u$ which can be written as the sum of a pair of pseudo-disjoint elements which are not disjoint. Then $G$ is not a group of divisibility.

Proof. Write $u=a+b$ with $a, b>0$ in $G$ as prescribed in the statement of the corollary, and suppose $0<c<a, b$. Then $\{a, b\}$ is a maximal pseudodisjoint set over $c$, and Theorem A applies.

Let $G$ be a p.o. group and $A$ be an $o$-ideal of $G$. We call $G$ a lex-extension of $A$ (by $G / A$ ) if for each $0<a \in A$ and $0<g \in G \backslash A, g>a$. $G$ is a direct lex-extension of $A$ if $A$ is a direct summand: equivalently, $G=B \oplus A$ and $0 \leqq g=(b, a)$ if and only if $b>0$, or $b=0$ and $a \geqq 0$. We then write $G=B \overrightarrow{\overrightarrow{\times}} A$. If $A$ and $B$ are $l$-groups then $G=B \overrightarrow{\times} A$ is a pseudo $l$-group [3], and under these assumptions $G$ is an l-group if and only if $A=0$ or $B$ is an $o$-group.

Call a weak order unit $u$ in an l-group $B$ decomposable if $u$ can be written as a sum of pairwise disjoint, strictly positive elements of $B$.

Corollary 2. Let $A \neq 0$ and $B$ be l-groups, and suppose that $B$ has a decomposable weak unit. Then $G=B \overrightarrow{\times} A$ is not a group of divisibility.

We compare our last corollary with Ohm's theorem 5.3 in [8]. Consider his condition labeled (5.1): there exist $b_{1}, b_{2} \in B$ such that $b_{1}$ and $b_{2}$ are

1 We may assume without loss of generality that the number 1 is in each $R_{\lambda}$. 
incomparable, and a subdirect representation of $B$ as a subdirect product of $o$-groups $B_{i}(i \in I)$ by an $l$-isomorphism $\sigma$ such that $b_{1} \sigma_{i} \neq b_{2} \sigma_{i}$, for all $i \in I$. It is equivalent to the existence of a decomposable weak order unit in $B$.

To see this note that if Ohm's (5.1) holds for an l-group $B$, and $b_{1}$ and $b_{2}$ are as specified above, then if we set $u=\left(b_{1}-b_{2}\right) \vee 0+\left(b_{2}-b_{1}\right) \vee 0$, $u$ is a decomposable weak order unit. For $u \sigma_{i}=\left(b_{1}-b_{2}\right) \sigma_{i} \vee 0+\left(b_{2}-b_{1}\right) \sigma_{i} \vee 0$, and so $u \sigma_{i}=\left(b_{1}-b_{2}\right) \sigma_{i}$ or $\left(b_{2}-b_{1}\right) \sigma_{i}$, either of which is $>0$. Hence $u$ is a weak order unit, and it is clearly decomposable.

Conversely, suppose $B$ has a decomposable weak order unit $u$, and $u=a+b$, with $0<a, b \in B$ and $a \wedge b=0$. If a minimal prime subgroup $N$ of $B$ contains $u$ then by the minimality of $N$ there exists an element $0<x \in B \backslash N$ such that $x \wedge u=0$, a contradiction. Consider then the family $\left\{N_{\lambda} \mid \lambda \in \Lambda\right\}$ of minimal prime subgroups of $B$; let $B_{\lambda}=B / N_{\lambda}$ and $\sigma: B \rightarrow \Pi B_{\lambda}$ be the induced $l$-embedding. Each $B_{\lambda}$ is an $o$-group and $u \sigma_{\lambda}>0$, for each $\lambda \in \Lambda$. Let $b_{1}=a-b$ and $b_{2}=0$; then this pair satisfies Ohm's condition relative to the mapping $\sigma$. (We refer the reader to [2, pp. 1.14-1.15 and pp. 2.13-2.14].)

His Theorem 5.3 is somewhat more general than Corollary 2 in view of the fact that we assume $A$ to be an l-group, whereas he does not.

Following Corollary 3.3 in [8] Ohm remarks that if one takes the polynomial ring $k[x, y]$ in two indeterminates over the field $k$, and localizes by the ideal generated by $x$ and $y$, one obtains a local ring whose group of divisibility is a cardinal sum of copies of $Z$, the integers in their usual order; the number of copies of $Z$ is at least 2 since the local ring is not a valuation ring. If $G$ is then the group of divisibility of a domain $D$ whose quotient field is $k$, Corollary 3.3 in [8] shows that the direct lex-extension of $G$ by this cardinal sum of integers is again a group of divisibility. If $G$ is an $l$-group such a lex-extension is a pseudo $l$-group which is not an l-group, providing a large class of examples of such pseudo l-groups which are groups of divisibility. ${ }^{2}$ In view of the observation in $\$ 1$ that every pseudo $l$-group can be embedded in a reasonably "nice" way in a $v$-group, the examples here contrasted with Theorem B leave a rather monstrous question mark as to the nature of groups of divisibility, not only in the context of pseudo l-groups, but in general as well.

3. Polynomial rings and Gauss' lemma. We conclude this note with a result that calculates for an integral domain $D$ whose group of divisibility is an l-group, the group of divisibility of its polynomial ring $D[x]$ in one variable. Curiously, an analogue of the classical Gauss lemma for

${ }^{2}$ In view of Theorem $A$ there are infinitely many copies of $Z$ in these cardinal sums. 
polynomials crops up at a rather crucial juncture. First, a general preliminary remark:

Proposition. Let $D$ be an integral domain, $G$ be its group of divisibility; then $G(D[x])$ is a direct extension of $G$ by a cardinal sum of copies of $Z$.

Proof. Let $k$ be the quotient field of $D$. We note here that the group of units $U(D)$ of $D$ is also the group of units of $D[x]$. Further $D[x]$ and $k[x]$ have same quotient field, namely $k(x)$, the field of rational functions in $x$ with coefficients in $k$. Finally, the group of units of $k[x]$ is $k^{*}$. Thus

$$
G=k^{*} / U(D), \quad G(D[x])=k(x)^{*} / U(D), \quad \text { and } \quad G(k[x])=k(x)^{*} / k^{*},
$$

and the latter is a cardinal sum of integers; see [7, Theorem 4.3]. Clearly, the inclusion of $G$ in $G(D[x])$ is a convex order embedding, and the canonical epimorphism $G(D[x]) \rightarrow G(k[x])$ is an o-epimorphism. Hence $G(D[x]) / G \simeq G(k[x])$; since $G(k[x])$ is abstractly a free abelian group, the extension is direct.

Now suppose $G=G(D)$ is an l-group; then $D$ has the following properties:

(1) any finite set of nonzero elements of $D$ has a greatest common divisor, and

(2) if $d$ divides $a b(a, b, d \in D)$ then $d=x y$ where $x$ divides $a$ and $y$ divides $b$. This is so because $G$, being an l-group, satisfies the Riesz interpolation property: if $0 \leqq a_{1}, a_{2} \in G$ and $0 \leqq b \in G$, then $b \leqq a_{1}+a_{2}$ implies that $b=b_{1}+b_{2}$, with $0 \leqq b_{i} \leqq a_{i}(i=1,2)$.

Call a polynomial $p(x)$ in $D[x]$ primitive if the greatest common divisor of the coefficients of $p(x)$ is a unit of $D$. If $G$ is an l-group any polynomial $g(x) \in D[x]$ can be written uniquely (up to units) as $g(x)=d \cdot g_{0}(x)$, where $g_{0}(x)$ is primitive and $d$ is the greatest common divisor of the coefficients of $g(x)$.

The following is a crucial lemma.

Lemma 3 (GAuss' Lemma). If the group of divisibility $G$ of an integral domain $D$ satisfies the Riesz interpolation property, the product of two primitive polynomials in $D[x]$ is primitive.

Proof. Let $p(x)=a_{0}+a_{1} x+\cdots+a_{m} x^{m}$ and $q(x)=b_{0}+b_{1} x+\cdots+$ $b_{n} x^{n}$ be primitive polynomials, and $p(x) q(x)=c_{0}+c_{1} x+\cdots+c_{m+n} x^{m+n}$. Suppose $d \in D$ divides all $c_{k}$, and is not a unit. Let $i_{0}\left(j_{0}\right)$ be the first index such that $d$ fails to divide $a_{i_{0}}\left(b_{j_{0}}\right)$; set $k_{0}=i_{0}+j_{0}$. Then $d$ divides $c_{k_{0}}=$ $a_{0} b_{k_{0}}+\cdots+a_{i_{0}} b_{j_{0}}+\cdots+a_{k_{0}} b_{0}$, and so $d$ divides $a_{i_{0}} b_{j_{0}}$. Since $G$ satisfies the Riesz interpolation property $d=x_{0} y_{0}$ where $x_{0}\left(y_{0}\right)$ divides $a_{i_{0}}\left(b_{j_{0}}\right)$. Now $x_{0}$ divides each $c_{k}$, each $a_{i}$ for $i=0,1, \cdots, i_{0}$ and each $b_{j}$ for $j=0$, $1, \cdots, j_{0}-1$. 
By induction, $x_{0}$ is a unit and so $d$ divides $b_{j_{0}}$, which is a contradiction. We conclude that $p(x) q(x)$ is primitive, and the lemma is proved.

Theorem C. If the group of divisibility $G$ of the integral domain $D$ is an l-group, then $G(D[x])$ is a cardinal sum of $G$ with a cardinal sum of copies of $Z$; in particular $G(D[x])$ is an l-group.

Proof. Recall that a saturated multiplicative system of an integral domain is a subset of nonzero elements, closed under multiplication, which contains along with an element $d$ all the divisors of $d$. Mott (see [7, Theorem 5.1]) showed that there is a natural isomorphism between the lattice of saturated multiplicative systems of an integral domain and the $o$-ideals of its group of divisibility.

Lemma 3 says that the subset $S$ of primitive polynomials in $D[x]$ is multiplicative; it is clearly saturated. Also, the nonzero elements of $D$ form a multiplicative system in $D[x]$ which is saturated; denote this subset by $D^{*}$. Since $G$ is an $l$-group we may write every nonzero polynomial $f(x)$ as a product of an element from $D^{*}$ and an element of $S$; evidently $S \cap D^{*}=U(D)$. By Mott's Theorem (and the logical extension thereof) there exist $o$-ideals $A$ and $B$ of $G(D[x])$ such that $G(D[x])$ is the cardinal sum of $A$ and $B$; if $A$ corresponds to $D^{*}$ then clearly $A \simeq G$, and it is immediate that $B$ (corresponding to $S$ ) is isomorphic to $G(k[x])$. This concludes the proof of Theorem C.

We offer the following remark in the way of a converse of Theorem C. Let $D$ be an integral domain and $G$ be its group of divisibility. Without any further assumptions $G$ is an $o$-ideal of $G(D[x])$; so suppose it splits off cardinally. Then $G(D[x])=G \prod M$, where $M$ is an $o$-ideal of $G(D[x])$; using Mott's correspondence again we come up with a saturated multiplicative system $T$ in $D[x]$ having the properties that (1) $D^{*} \cap T=U(D)$ and (2) every nonzero polynomial $f(x)$ can be written (uniquely up to units) as the product of an element of $D^{*}$ and one from $T$. Now let $S$ be the set of primitive polynomials; clearly $S \subseteq T$, and if $p(x) \in T$ but is not primitive, then write $p(x)=d \cdot q(x)$, and pick $d$ to be a nonunit of $D$. Since $T$ is saturated $q(x) \in T$, but this violates the uniqueness of such expressions. Hence $T=S$.

Moreover pick $0 \neq a, b \in D$ and consider $f(x)=a+b x$; by writing $f(x)$ as a product of an element from $D^{*}$ and an element from $S$ we locate the greatest common divisor of $a$ and $b$. We can therefore make the following conclusion.

THEOREM D. Let $G$ be the group of divisibility of the integral domain $D$; let $H=G(D[x])$. If $H$ is the cardinal sum of $G$ and $G(k[x])$ then

(1) any finite set of nonzero elements of $D$ has a greatest common 
divisor, and

(2) the subset $S$ of primitive polynomials over $D$ is a saturated multiplicative system.

If $G$ satisfies the Riesz interpolation property it is an l-group.

Finally, in view of Theorem C conditions (1) and (2) are sufficient to insure that $G$ split as a cardinal summand of $H$.

In closing we pose one of many questions that arise naturally here: if $G=G(D)$ satisfies the Riesz interpolation property, then does $G(D[x])$ ?

\section{BiBLIOGRAPHY}

1. P. Conrad, Representations of partially ordered abelian groups as groups of real valued functions, Acta Math. 116 (1966), 199-221. MR 34 \#1418.

2. — Lattice ordered groups, Tulane University, New Orleans, La., 1970.

3. P. Conrad and J. R. Teller, Abelian pseudo lattice ordered groups (preprint).

4. P. Jaffard, Contribution à l'étude des groupes ordonnés, J. Math. Pures Appl. (9) 32 (1953), 203-280. MR 15, 284.

5. - Les systèmes d'idéaux, Travaux et Recherches Mathematiques, IV, Dunod, Paris, 1960. MR 22 \#5628.

6. W. Krull, Allgemeine Bewertungstheorie, J. Reine Angew. Math. 167 (1931), 160-196.

7. J. Mott, The group of divisibility and its applications (preprint).

8. J. Ohm, Semi-valuations and groups of divisibility, Canad. J. Math. 21 (1969), 576-591. MR 39 \#4146.

9. D. Zelinsky, Topological characterizations of fields with valuations, Bull. Amer. Math. Soc. 54 (1948), 1145-1150. MR 10, 426.

Department of Mathematics, University of Florida, Gainesville, Florida 32601 